\title{
SIWF-Award: besonderes Engagement für die Weiterbildung
}

\section{Werner Bauer ${ }^{a}$, Raphael Stolz ${ }^{b}$, Nadja Jenni ${ }^{c}$}

${ }^{a}$ Dr. med., Präsident des Schweizerischen Instituts für ärztliche Weiter- und Fortbildung SIWF; b Dr. med., Vizepräsident des Schweizerischen Instituts für ärztliche Weiter- und Fortbildung SIWF; ' M.Sc., Wissenschaftliche Mitarbeiterin SIWF/FMH

Hat sich ein früherer Weiterbildner exemplarisch für Ihre Weiterbildung eingesetzt? Haben Sie dank der hohen didaktischen Kompetenz einer Weiterbildnerin speziell erfreuliche Fortschritte in Bezug auf Kenntnisse und Fähigkeiten machen können? Dann nominieren Sie diese engagierten Weiterbildungsverantwortlichen für den SIWF-Award für besonderes Engagement in der Weiterbildung!

Wir freuen uns, bereits zum vierten Mal die Ausschreibung für den SIWF-Award veröffentlichen zu können. Die zahlreichen Nominationen und das positive Echo haben uns bestätigt, dass eine solche Auszeichnung sinnvoll ist und ihren Zweck erfüllt. Die Verleihung ist deshalb fester Bestandteil der SIWFAgenda geworden.

Entscheidende Grundlage für eine optimale Vermittlung von Können und Wissen an die Weiterzubildenden ist das Engagement der Kaderärztinnen und -ärzte. Diese Aufgabe lässt sich nur beschränkt durch Pflichtenhefte definieren, viel wichtiger sind persönliches Engagement und Begeisterung. Die Belastungen in der medizinischen Arbeitswelt sind vielfältig, und die zeitlichen sowie finanziellen Ressourcen werden stets knapper, umso mehr sollten besonders aktive und motivierte Weiterbildnerinnen und Weiterbildner auch Anerkennung erhalten. Das SIWF bietet aus diesem Grund Assistenzärztinnen und -ärzten die Möglichkeit, dem ausserordentlichen Engagement solcher Weiterbildungsverantwortlichen explizit Ausdruck zu verleihen, ohne aber eine Rangliste erstellen zu wollen.

\section{Nominierung durch Assistenzärztinnen und -ärzte}

Für den SIWF-Award können Personen nominiert werden, welche zurzeit in der ärztlichen Weiterbildung aktiv tätig sind. Im Fokus stehen Kaderärztinnen und -ärzte, welche sich persönlich für die Weiterbildung von angehenden Fachärzten und -ärztinnen einsetzen und besonders kompetent und initiativ bei der Weitergabe von Kenntnissen und Fertigkeiten mitwirken. Nominationsberechtigt sind Ärztinnen und Ärzte, die sich zurzeit in der Weiterbildung zu einem Facharzttitel befinden oder vor weniger als einem Jahr den Facharzttitel erworben haben. Eine Nomination ist dann gültig, wenn sie durch zwei Personen gemeinsam erfolgt. Sie soll die persönliche Wertschätzung für die wahrgenommene Weiterbildungsqualität und für das Engagement der Weiterbildungsverantwortlichen ausdrücken. Damit aufgrund des Nominationsprozesses keine Vorteile oder Konflikte am Arbeitsplatz entstehen können, dürfen nur Weiterbildungsverantwortliche nominiert werden, bei welchen die Nominierenden aktuell nicht mehr angestellt sind. Die Namen der nominierenden Personen werden nicht veröffentlicht und den Nominierten auch nicht mitgeteilt. Es wird keine «Rangliste» der Nominierten erstellt.

\section{Jetzt Weiterbildungsverantwortliche} nominieren!

Der SIWF-Award gibt die Möglichkeit, besonders engagierten und kompetenten ärztlichen Weiterbildungsverantwortlichen eine Anerkennung auszudrücken. Hat ein ehemaliger Weiterbildner oder eine ehemalige Weiterbildnerin bei Ihnen einen bleibenden Eindruck hinterlassen? Dann nominieren Sie ihn oder sie für den SIWF-Award.

Senden Sie Ihr Nominationsschreiben bitte in elektronischer Form an siwf[at]fmh.ch mit dem Vermerk «SIWF-Award für besonderes Engagement in der Weiterbildung".

Einsendeschluss: 6. August 2017

Weitere Informationen finden Sie auf www.siwf.ch. Wenn Sie Fragen haben, erreichen Sie uns über siwf[at]fmh.ch oder unter 0313591111 


\section{Jetzt Nomination einreichen!}

Korrespondenz: Schweizerisches Institut für ärztliche Weiter- und Fortbildung SIWF FMH

Elfenstrasse 18

Postfach 300

CH-3000 Bern 15

Tel. 0313591111

siwf[at]fmh.ch
Um jemanden zu nominieren, können Sie das vorgesehene Formular herunterladen unter www.siwf.ch $\rightarrow$ SIWF $\rightarrow$ Weiterbildung $\rightarrow$ Allgemein $\rightarrow$ SIWFAward. Das ausgefüllte Gesuchsformular senden Sie bitte bis 6. August 2017 in elektronischer Form an: siwf[at]fmh.ch

Die SIWF-Geschäftsleitung überprüft, ob die Nominierung formell korrekt ist, und entscheidet abschlies- send über die Gültigkeit der einzelnen Nominationen. Alle korrekt Nominierten erhalten als Würdigung ihres Engagements in der Weiterbildung eine Anerkennungsurkunde und ein Präsent. Sie werden (nach Rückfrage) auf der SIWF-Website (www.siwf.ch) aufgeführt und am MedEd-Symposium vom 20. September 2017 namentlich genannt.
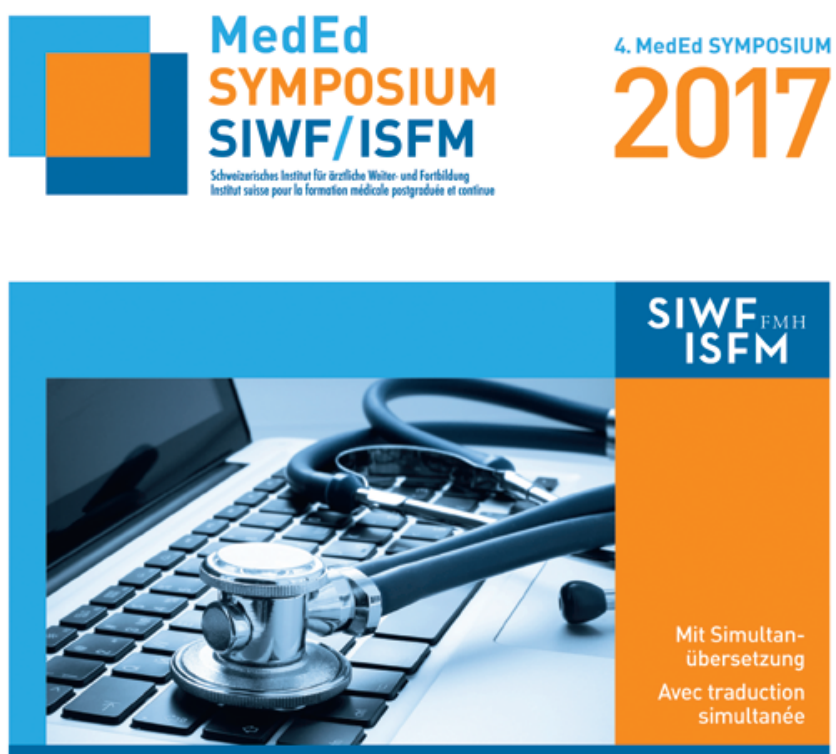

Perspektiven der ärztlichen Bildung Perspectives de la formation médicale

Das MedEd-Symposium ist im Rahmen der erweiterten Fortbildun hallen Fachgebieten mit $\mathbf{7}$ Credits anerkannt (SIWF-approved. Symposium MedEd donne droit à 7 crédits dans toutes les disciplines 